\title{
On the Honesty in Nonlocal and Discrete Fragmentation Dynamics in Size and Random Position
}

\author{
S. C. Oukouomi Noutchie and E. F. Doungmo Goufo \\ Department of Mathematical Sciences, North-West University, Mafikeng 2735, South Africa \\ Correspondence should be addressed to E. F. Doungmo Goufo; franckemile2006@yahoo.ca
}

Received 19 April 2013; Accepted 22 May 2013

Academic Editors: M. Escobedo and K. Ezzinbi

Copyright (C) 2013 S. C. Oukouomi Noutchie and E. F. Doungmo Goufo. This is an open access article distributed under the Creative Commons Attribution License, which permits unrestricted use, distribution, and reproduction in any medium, provided the original work is properly cited.

\begin{abstract}
A discrete initial-value problem describing multiple fragmentation processes, where the fragmentation rate is size and position dependent and where new particles are spatially randomly distributed according to some probabilistic law, is investigated by means of parameter-dependent operators together with the theory of substochastic semigroups with a parameter. The existence of semigroups is established for each parameter and "glued" together so as to obtain a semigroup to the full space. Under certain conditions on each fragmentation rate, we used Kato's Theorem in $L_{1}$ to show the existence of the generator and we provide sufficient conditions for honesty.
\end{abstract}

\section{Introduction}

The process of fragmentation of clusters occurs in numerous domains of pure and applied sciences, such as the depolymerization, the rock fractures, and break of droplets. The fragmentation rate can be size and position dependent, and new particles resulting from the fragmentation are spatially randomly distributed according to some probability density. When it is supposed that every group of size $n \in \mathbb{N}$ (one $n$-group) in a system of particles clusters consists of $n$ identical fundamental units (monomers), then the mass of every group is simply a multiple positive integer of the mass of the monomer. We focus here on clusters that are discrete; that is, they consist of a finite number of elementary (unbreakable) particles which are assumed to be of unit mass. The state at a given time $t$ is the repartition at that time of all aggregates according to their size $n$ and their position $x$. The evolution of such particle-mass-position distribution is given by an integrodifferential [1] equation as we will see in this paper.

Before going farther let us review what have already been done. Various types of fragmentation equations have been comprehensively analyzed in numerous works (see, e.g., [2-9]). Conservative and nonconservative regimes for fragmentation equations have been thoroughly investigated, and, in particular, the breach of the mass conservation law (called shattering) has been attributed to a phase transition creating a dust of zero-size particles with nonzero mass, which are beyond the model resolution. Shattering can be interpreted from the probabilistic point of view as the explosion in the Markov process describing fragmentation $[8,10]$ and from an analytic point of view as dishonesty of the semigroup associated with the model $[2,7]$. KineticType Models with Diffusion were investigated in [11] where the author showed that the diffusive part does not affect the breach of the conservation laws. But discrete fragmentation processes have not widely been investigated yet. In [1], a discrete model with the concentration depending only on the size $n$ of clusters and time $t$ is analyzed and the author used compactness of the semigroups to analyze their long time behavior and proved that they have the asynchronous growth property.

In this paper, we exploit a technique called the method of semigroups with a parameter [2] to analyze discrete fragmentation models with the concentration of particles depending not only on the size $n$ of clusters and time $t$, but also on the random position $x$ of the clusters in the space. 


\section{Models' Description and Assumptions}

We focus on models with discrete size; that is, we assume that the mass of a particle can be an arbitrary positive integer. The solution $p_{n}=p(t, x, n)$ which characterizes the state of the system at any moment $t$ is the particle-mass-position distribution defined as $p: \mathbb{R}_{+} \times \mathbb{R}^{3} \times \mathbb{N} \rightarrow \mathbb{R}_{+}$. For the reasons of simplicity, we will sometimes use $p(t, x, n)=p_{n}(t, x)$ or simply $p_{n}$. During the unit time, a fraction $a_{n}(x)=a(x, n)$ (or simply $a_{n}$ ) of aggregates of size $n$ and located at $x$ are undergoing breakup. We assume that for each $n \in \mathbb{N}$, there are two constants $0<\theta_{1}$ and $\theta_{2}$ such that

$$
\theta_{1} \alpha_{n} \leq a_{n}(x) \leq \theta_{2} \alpha_{n}
$$

with $\alpha_{n} \in \mathbb{R}_{+}$and independent of the state variable $x$. Clearly we require that

$$
a_{1}(x)=0
$$

for every $x \in \mathbb{R}^{3}$, since a cluster of size one cannot split.

Once a group of size $m$ and position $x$ breaks, the expected average number of $n$-group produced upon the splitting is a nonnegative measurable function $b_{n, m}(x)=$ $b(x, n, m)$ defined on $\mathbb{R}^{3} \times \mathbb{N} \times \mathbb{N}$ with support in the set

$$
\mathbb{R}^{3} \times\{(n, m) \in \mathbb{N} \times \mathbb{N}: m>n\}
$$

The sum of all individuals obtained by fragmentation of an $n$-group should again be $n$ hence it follows that for any $n \in \mathbb{N}$, $x \in \mathbb{R}^{3}$

$$
\sum_{m=1}^{n-1} m b(x, m, n)=n
$$

Since a group of size $m \leq n$ cannot split to form a group of size $n$, we require that

$$
b_{n, m}=0 \quad \forall m \leq n .
$$

Furthermore the expected number of daughter particles produced by fragmentation of an $n$-group (with position $x$ ) is, by definition, given by $\sum_{m=1}^{n-1} b(x, m, n)$.

After cluster fragmentation, new originating groups have different centers distributed according to a given probabilistic law $h(\cdot, n, m, y)$. This is the probability density that after a breakup of an $m$-aggregate (with the center at $y$ ), the new formed $n$-group will be located at $x$. Therefore

$$
\int_{\mathbb{R}^{3}} h(x, n, m, y) d x=1
$$

The equation describing the evolution of the particle-masssize distribution function for a discrete system undergoing fragmentation can be derived by balancing loss and gain of clusters of size $n$ (with position $x$ ) over a short period of time and is given by

$$
\begin{aligned}
\frac{\partial p}{\partial t}(t, x, n)= & -a_{n}(x) p(t, x, n) \\
& +\sum_{m=n+1}^{\infty} \int_{\mathbb{R}^{3}} a_{m}(y) b_{n, m}(y) h(x, n, m, y) p \\
& \quad \times(t, y, m) d y, \quad n=1,2,3, \ldots,
\end{aligned}
$$

where in terms of $n$ and $x$, the state of the system is characterized at any moment $t$ by the density (or concentration) of particles $p(t, x, n)$.

\section{Well Posedness of the Fragmentation Problem}

Since $p_{n}=p(t, x, n)$ is the density of groups of size $n$ at the position $x$ and mass is expected to be a conserved quantity, the most appropriate space to work in is the space

$$
\begin{gathered}
\mathscr{X}_{1}:=\left\{\mathbf{g}=\left(g_{n}\right)_{n=1}^{\infty}: \mathbb{R}^{3} \times \mathbb{N} \ni(x, n) \longrightarrow g_{n}(x),\right. \\
\left.\|\mathbf{g}\|_{1}:=\int_{\mathbb{R}^{3}} \sum_{n=1}^{\infty} n\left|g_{n}(x)\right| d x<\infty\right\} .
\end{gathered}
$$
bution

We complement (7) with the initial mass-position distri-

$$
p(0, x, n)=\stackrel{o}{p}_{n}(x), \quad n=1,2,3, \ldots
$$

In $\mathscr{X}_{1}$, we can rewrite (7) and (9) in more compact form:

$$
\begin{gathered}
\frac{\partial}{\partial t} \mathbf{p}=\mathfrak{A} \mathbf{p}+\mathfrak{B} \mathbf{p}, \\
\mathbf{p}_{t=0}=\mathbf{p}
\end{gathered}
$$

Here $\mathbf{p}$ is the vector $(p(t, x, n))_{n \in \mathbb{N}}, \mathfrak{A}$ is the diagonal matrix $\left(a_{n}(x)\right)_{n \in \mathbb{N}}, \mathfrak{B}$ is defined by the expression

$\mathfrak{B p}$

$$
=\left(\sum_{m=n+1}^{\infty} \int_{\mathbb{R}^{3}} a_{m}(y) b_{n, m}(y) h(x, n, m, y) p_{m}(y) d y\right)_{n=1}^{\infty},
$$

and $\stackrel{\mathbf{o}}{\mathbf{p}}$ is the initial vector $\left(\stackrel{o}{p_{n}}(x)\right)_{n \in \mathbb{N}}$ which belongs to $\mathscr{X}_{1}$. We introduce operators $\mathbf{A}$ and $\mathbf{B}$ in $\mathscr{X}_{1}$ defined by

$$
\begin{gathered}
{[\mathbf{A p}](x, n)=[\mathfrak{A} \mathbf{p}](x, n), \quad D(\mathbf{A})=\left\{\mathbf{g} \in \mathscr{X}_{1} ; a \mathbf{g} \in \mathscr{X}_{1}\right\},} \\
{[\mathbf{B} \mathbf{p}](x, n)=[\mathfrak{B} \mathbf{p}](x, n), \quad D(\mathbf{B}):=D(\mathbf{A}) .}
\end{gathered}
$$


Lemma 1. $(\mathbf{A}+\mathbf{B}, D(\mathbf{A}))$ is a well-defined operator.

Proof. We need to show that $\mathbf{B} D(\mathbf{A}) \subset \mathscr{X}_{1}$. For every $\mathbf{g} \in$ $D(\mathbf{A})$,

$$
\begin{aligned}
\|\mathbf{B g}\|_{1} & =\int_{\mathbb{R}^{3}}\left(\sum_{n=1}^{\infty} n \sum_{m=n+1}^{\infty} \int_{\mathbb{R}^{3}} a_{m}(y) b_{n, m}(y) h(x, n, m, y)\right. \\
& \times|g(y, m)| d y) d x \\
& =\int_{\mathbb{R}^{3}}\left(\sum_{n=1}^{\infty} n \sum_{m=n+1}^{\infty} a_{m}(y) b_{n, m}(y)|g(y, m)|\right) d y \\
& =\int_{\mathbb{R}^{3}} \sum_{m=2}^{\infty} a_{m}(y)|g(y, m)|\left(\sum_{n=1}^{\infty} n b_{n, m}(y)\right) d y \\
& =\int_{\mathbb{R}^{3}} \sum_{m=2}^{\infty} a_{m}(y)|g(y, m)|\left(\sum_{n=1}^{m-1} n b_{n, m}(y)\right) d y \\
& =\int_{\mathbb{R}^{3}} \sum_{m=2}^{\infty} m a_{m}(y)|g(y, m)| d y \\
& =\int_{\mathbb{R}^{3}} \sum_{m=1}^{\infty} m a_{m}(y)|g(y, m)| d y \\
& =\| \mathbf{A g}_{1} \\
& <\infty^{\infty},
\end{aligned}
$$

where we have used (6), (4), and (5), respectively. Then $\|\mathbf{B g}\|_{1}=\|\mathbf{A g}\|_{1} \forall \mathbf{g} \in D(\mathbf{A})$, so that we can take $D(\mathbf{B}):=D(\mathbf{A})$, and $(\mathbf{A}+\mathbf{B}, D(\mathbf{A}))$ is well defined.

3.1. Mathematical Setting and Analysis. We note that the operators on the left-hand side of (12) have the property that one of the variables is a parameter and, for each value of this parameter, the operator has a certain desirable property (like being the generator of a semigroup) with respect to the other variable. Thus we need to work with parameter-dependent operators that can be "glued" together in such a way that the resulting operator inherits the properties of the individual ones. Let us provide a framework for such a technique called the method of semigroups with a parameter [2].

Let $\Lambda=\mathbb{R}^{3} \times \mathbb{N}$ and consider the space $\mathscr{X}:=L_{p}(V, X)$ where $1 \leq p<\infty,(V, d m)$ is a measure space and $X$ a Banach space. Let us suppose that we are given a family of operators $\left\{\left(A_{v}, D\left(A_{v}\right)\right)\right\}_{v \in V}$ in $X$ and define the operator $(\mathbb{A}, D(\mathbb{A}))$ acting in $\mathscr{X}$ according to the following formulae:

$$
\begin{gathered}
\mathscr{D}(\mathbb{A}):=\left\{g \in \mathscr{X} ; g(v) \in D\left(A_{v}\right)\right. \text { for almost every } \\
v \in V, \mathbb{A} g \in \mathscr{X}\},
\end{gathered}
$$

and, for $g \in \mathscr{D}(\mathbb{A})$,

$$
(\mathbb{A g})(v):=A_{v} g(v)
$$

for every $v \in \mathscr{X}_{1}$. We have the following proposition.
Proposition 2 (see [2, Proposition 3.28]). If for almost any $v \in V$ the operator $A_{v}$ is $m$-dissipative in $X$, and the function $v \rightarrow R\left(\lambda, A_{v}\right) g(v)$ is measurable for any $\lambda>0$ and $g \epsilon$ $\mathscr{X}$, then the operator $\mathbb{A}$ is an $m$-dissipative operator in $\mathscr{X}$. If $\left(G_{v}(t)\right)_{t \geq 0}$ and $(\mathscr{G}(t))_{t \geq 0}$ are the semigroups generated by $A_{v}$ and $\mathbb{A}$, respectively, then for almost every $v \in V, t \geq 0$, and $g \in \mathscr{X}$ we have

$$
[\mathscr{G}(t) g](v):=G_{v}(t) g(v)
$$

Using the above ideas we introduce operators that cover the case of the present applications. Here the $n$ variable is the parameter and $x$ is the main variable. We set

$$
X_{x}:=L_{1}\left(\mathbb{R}^{3}, d x\right):=\left\{\psi:=\|\psi\|=\int_{\mathbb{R}^{3}}|\psi(x, n)| d x<\infty\right\}
$$

and define in $X_{x}$ the operators $\left(\mathscr{A}_{n}, D\left(\mathscr{A}_{n}\right)\right)$ as

$$
\begin{gathered}
\mathscr{A}_{n} p(t, x, n)=a_{n}(x) p(t, x, n), \\
D\left(\mathscr{A}_{n}\right):=\left\{p_{n} \in X_{x}, \mathscr{A}_{n} p_{n} \in X_{x}\right\}, \quad n \in \mathbb{N} .
\end{gathered}
$$

Using the above proposition, we can take $\mathbb{A}=\mathbf{A}, \mathscr{X}=\mathscr{X}_{1}=$ $L_{1}\left(\mathbb{N}, X_{x}\right)=L_{1}(\Lambda, d \mu d \varsigma)=L_{1}\left(\mathbb{R}^{3} \times \mathbb{N}, d \mu d \varsigma\right)$, where $\mathbb{N}$ is equipped with the counting measure $d \varsigma$ and $d \mu=d x$ is the Lebesgue measure in $\mathbb{R}^{3}$. In the notation of the proposition, $(\mathbb{N}, d \varsigma)=(V, d m), X_{x}=X$, and $A_{v}=\mathscr{A}_{n}$; therefore $\left(\mathscr{A}_{n}, D\left(\mathscr{A}_{n}\right)\right)_{n \in \mathbb{N}}$ is a family of operators in $X_{x}$, and using (15), we have

$$
(\mathrm{Ap})_{n}:=\mathscr{A}_{n} p_{n}
$$

Theorem 3. There is an extension $K$ of $\mathbf{A}+\mathbf{B}$ that generates a positive semigroup of contractions $\left(S_{K}(t)\right)_{t \geq 0}$ on $\mathscr{X}_{1}$. Moreover, for each $\stackrel{\mathbf{o}}{\mathbf{p}}=\left(\stackrel{o}{p}_{n}(x)\right)_{n \in \mathbb{N}} \in D(K)$ there is a measurable representation $\mathbf{p}$ of $S_{K}(t) \stackrel{\mathbf{o}}{\mathbf{p}}$ which is absolutely continuous with respect to $t \geq 0$ for almost any $(x, n)$ and such that (10) is satisfied almost everywhere.

Proof. To prove the first part of the theorem, let's show that for each $n \in \mathbb{N}, \mathscr{A}_{n}$ generates a positive semigroup of contractions. This assertion follows from (1) and the fact that the operator $\mathscr{A}_{n}$ is a multiplication operator on $X_{x}$ induced by the measurable function $a$; then, it is closed and densely defined [12]. Also for any $\lambda>0$, it is obvious that $\lambda I-\mathscr{A}_{n}$ is bijective and the resolvent $R\left(\lambda, \mathscr{A}_{n}\right)$ of $\mathscr{A}_{n}$ satisfies the estimate

$$
\left\|R\left(\lambda, \mathscr{A}_{n}\right) \psi\right\| \leq \frac{1}{\lambda}\|\psi\|
$$

for any $\psi \in X_{x}$. Furthermore for any positive $\lambda$, the operator $R\left(\lambda, \mathscr{A}_{n}\right)$ is nonnegative. Therefore $\left(\mathscr{A}_{n}, D\left(\mathscr{A}_{n}\right)\right)$ generates a positive semigroup of contractions. Thus, using relation (16) of Proposition 2, we claim that $(\mathbf{A}, D(\mathbf{A}))$ also generates a positive semigroup of contractions. 
It is clear that $(\mathbf{B}, D(\mathbf{B}))$ is positive. Furthermore for any $\mathbf{p} \in D(\mathbf{A})$, by the calculations in the Lemma 1, we have $\|\mathbf{A g}\|_{1}=\|\mathbf{B g}\|_{1}$. Then

$$
\begin{aligned}
& \int_{\Lambda}(-\mathbf{A} \mathbf{p}+\mathbf{B} \mathbf{p}) d \mu d \varsigma \\
&=-\int_{\mathbb{R}^{3}} \sum_{m=1}^{\infty} m a_{m}(y)|g(y, m)| d y \\
& \quad+\int_{\mathbb{R}^{3}}\left(\sum_{n=1}^{\infty} n \sum_{m=n+1}^{\infty} \int_{\mathbb{R}^{3}} a_{m}(y) b_{n, m}(y) h(x, n, m, y)\right. \\
&\quad \times|g(y, m)| d y) d x
\end{aligned}
$$$$
=0 \text {. }
$$

Thus the assumptions of Kato's Theorem in $L_{1}$ (see [2, Corollary 5.17]) are satisfied. Therefore there is an extension $K$ of $\mathbf{A}+\mathbf{B}$ generating a substochastic semigroup $\left(G_{K}(t)\right)_{t \geq 0}$. Let $K_{n}$ be the $n$th of $K$ according to (19) and Proposition 2; then from (16), it suffices to prove the assertions of the theorem for each $K_{n}, n \in \mathbb{N}$. For any $\stackrel{o}{p}_{n} \in D\left(K_{n}\right)$, the function $t \rightarrow G_{K_{n}}(t) \stackrel{o}{p_{n}}$ is a $C^{1}$-function in the norm of $X_{x}$ and satisfies the equation

$$
\frac{d}{d t} G_{K_{n}}(t) \stackrel{o}{p_{n}}=K_{n} G_{K_{n}}(t) \stackrel{o}{p_{n}}
$$

where the equality holds for any $t>0$ in the sense of equality in $X_{x}$. The initial condition is satisfied in the following sense:

$$
\lim _{t \rightarrow 0^{+}} G_{K_{n}}(t) \stackrel{o}{P_{n}}=\stackrel{o}{p_{n}}
$$

where the convergence is in the $X_{x}$-norm.

In order to prove the second part of this theorem we make use of the theory of extensions and the theory of $L$ spaces [2]. Let $\Theta$ be the set of finite almost everywhere measurable functions defined on $\mathbb{R}^{3}$. We recall that $\Theta$ is a lattice with respect to the usual relation ( $\leq$ almost everywhere), $X_{x} \subset \Theta$, and $X_{x}$ is a sublattice of $\Theta$. We denote by $\left(X_{x}\right)_{+}$and $\Theta_{+}$ the positive cones of $X_{x}$ and $\Theta$, respectively. For each $n \in$ $\mathbb{N}$ we introduce the operator $D_{n}$ defined such that for any nondecreasing sequence $\left(\psi_{k}\right)_{k \in \mathbb{N}}$ in $\left(X_{x}\right)_{+}$with $\sup _{k \in \mathbb{N}} \psi_{k}=$ $\psi \in \Theta_{+}$,

$$
D_{n} \psi:=\sup _{k \in \mathbb{N}} \mathscr{B}_{n} \psi_{k}
$$

where $\mathscr{B}_{n}$ is given by $\mathfrak{B} \mathbf{p}=\left(\mathscr{B}_{n}\right)_{n=1}^{\infty}$ defined in (11). Since $\mathscr{B}_{n}$ is an integral operator with positive kernel, Lebesgue's monotone convergence theorem yields that $D_{n}=\mathscr{B}_{n}$. Thus, [2, Theorem 6.20] yields $K_{n} \subset \mathscr{A}_{n}+\mathscr{B}_{n}$. Hence $G_{K_{n}}(t) \stackrel{o}{p_{n}}$ satisfies

$$
\begin{aligned}
{\left[\frac{d}{d t} G_{K_{n}}(t) \stackrel{o}{p_{n}}\right](x, n)=} & {\left[\mathscr{A}_{n} G_{K_{n}}(t) \stackrel{o}{p_{n}}\right](x, n) } \\
& +\left[\mathscr{B}_{n} G_{K_{n}}(t) \stackrel{o}{p_{n}}\right](x, n),
\end{aligned}
$$

for each fixed $t>0$, where the right-hand side does not depend (in the sense of equality almost everywhere) on what representation of the solution $G_{K_{n}}(t) \stackrel{o}{p_{n}}$ is considered. Making use of the fact that $X_{x}$ is an $L$ space, from [13, Theorem 3.4.2], we have that since the function $G_{K_{n}}(t) \stackrel{o}{p_{n}}$ is strongly differentiable, there is a representation $p(t, x, n)=$ $p_{n}$ of $G_{K_{n}}(t) \stackrel{o}{p_{n}}$ that is absolutely continuous with respect to $t \geq 0$ for almost any $(x, n) \in \mathbb{R}_{+} \times \mathbb{R}^{3}$, and that satisfies $(\partial / \partial t) p(t, x, n)=\left[(d / d t) G_{K_{n}}(t) \stackrel{o}{p_{n}}\right](x, n)$ for any $t \geq 0$ and almost any $(x, n)$. Hence, taking this representation, we obtain that

$$
\begin{aligned}
\frac{\partial p}{\partial t}(t, x, n) \\
=-a_{n}(x) p_{n} \\
\quad+\sum_{m=n+1}^{\infty} \int_{\mathbb{R}^{3}} a_{m}(y) b_{n, m}(y) h(x, n, m, y) p_{m}(y) d y
\end{aligned}
$$

holds almost everywhere on $\mathbb{R}_{+} \times \mathbb{R}^{3}$. Moreover, the continuity of $p_{n}$ with respect to $t$ for almost every $(x, n)$ shows that $\lim _{t \rightarrow 0^{+}} p_{n}=\bar{p}(x, n)$ exists almost everywhere. From (23) we see that there is a sequence $\left(t_{k}\right)_{k \in \mathbb{N}}$ converging to 0 such that $\lim _{k \rightarrow \infty} p\left(t_{k}, x, n\right)=\stackrel{o}{p_{n}}(x, n)$, for almost every $(x, n)$. Here we can use the same representation as above because we are dealing with a (countable) sequence. Indeed, changing the representation on a set of measure zero for each $n$ and further taking the union of all these sets still produce a set of measure zero. Thus $\stackrel{o}{p}_{n}=\bar{p}_{n}$ almost everywhere.

In general for each $n \in \mathbb{N}$, the function $G_{K_{n}}(t) \stackrel{o}{p_{n}}$ is not differentiable if $\stackrel{o}{p}_{n} \in X_{x} \backslash D\left(K_{n}\right)$. Therefore it cannot be a classical solution of the Cauchy problem (22) and (23). However it is a mild solution, that is, it is a continuous function such that $\int_{0}^{t} p_{n}(\tau) d \tau \in D\left(K_{n}\right)$ for any $t \geq 0$, satisfying the integrated version of (22) and (23):

$$
p_{n}(t)=\stackrel{o}{p}_{n}+K_{n} \int_{0}^{t} p_{n}(\tau) d \tau
$$

Corollary 4. If $\stackrel{o}{p}_{n} \in X_{x} \backslash D\left(K_{n}\right)$, then $p_{n}=\left[G_{K_{n}}(t) \stackrel{o}{p_{n}}\right](x, n)$ satisfies the equation

$$
\begin{aligned}
p(t, x, n)= & \stackrel{o}{p}{ }_{n}(x, n)-a_{n}(x) \int_{0}^{t} p(\tau, x, n) d \tau \\
& +\sum_{m=n+1}^{\infty} \int_{\mathbb{R}^{3}} a_{m}(y) b_{n, m}(y) h(x, n, m, y) p_{m}(y) \\
& \times\left(\int_{0}^{t} p(\tau, y, n) d \tau\right) d y .
\end{aligned}
$$


Proof. Because $p_{n}$ is continuous in the norm of $X_{x}=$ $L_{1}\left(\mathbb{R}^{3}, d x\right)$, we can use the fact that $X_{x}$ is of type $L$ (see [2, Theorem 2.39]) to claim that $a a_{n}(x) \int_{0}^{t} p(\tau, x, n) d \tau$ is defined for almost any $(x, n)$ and any $t$, and hence we can write

$$
\begin{aligned}
& {\left[\left(\mathscr{A}_{n}+\mathscr{B}_{n}\right) \int_{0}^{t} p(\tau) d \tau\right](x, n)} \\
& =-a_{n}(x) \int_{0}^{t} p(\tau, x, n) d \tau \\
& +\sum_{m=n+1}^{\infty} \int_{\mathbb{R}^{3}} a_{m}(y) b_{n, m}(y) h(x, n, m, y) p_{m}(y) \\
& \quad \times\left(\int_{0}^{t} p(\tau, y, n) d \tau\right) d y .
\end{aligned}
$$

Thus, combining the result used in the previous theorem that $K_{n} \subset \mathscr{A}_{n}+\mathscr{B}_{n}$ with (27) we obtain (28).

Next we provide a fairly general condition for honesty of $\left(G_{K_{n}}(t)\right)_{t \geq 0}$.

\section{Honesty}

Because the total number of individuals in a population is not modified by interactions (fragmentation) among groups, the following conservation law is supposed to be satisfied throughout the evolution:

$$
\frac{d}{d t} U(t)=0
$$

where $U(t)=\sum_{n=1}^{\infty} \int_{\mathbb{R}^{3}} n p(t, x, n) d x=\sum_{n=1}^{\infty} n \int_{\mathbb{R}^{3}} p(t, x, n) d x$ is the total number of particles (total mass) in the system. This is formally expressed by (7) as the mass rate equation can be found by multiplying (7) by $n$, integrating over $\mathbb{R}^{3}$, summing from $n=1$ to $\infty$ and using (4), which agrees with the physics of the process as fragmentation should simply rearrange the distribution of masses of the particles without altering the total mass of the system. However, the validity of (30) depends on certain properties of the solution $p$ that we tacitly assumed during the integration and which are far from obvious. In fact, by analyzing models with specific coefficients, several authors have observed that the local version of (30) is not valid [9]. In other words, there occurs an unexpected mass loss in the system. In local fragmentation, the unaccounted for mass loss was termed shattering fragmentation and was attributed to the phase transition in which a dust of particles with zero size and nonzero mass is formed. The presence of $x$ in (30) suggests that honesty in nonlocal discrete fragmentation depends also on the spatial properties of the fragmentation kernels. In this section we provide sufficient conditions for the discrete fragmentation semigroup to be honest for general coefficients.
Lemma 5. Assume that for any $\mathbf{p}=\left(p_{n}\right)_{n=1}^{\infty} \in\left(X_{1}\right)_{+}$such that $-\mathbf{A p}+\mathbf{B p} \in \mathscr{X}_{1}$ we have the inequality

$$
\int_{\Lambda}(-\mathbf{A p}+\mathbf{B} \mathbf{p}) d \mu d \varsigma \geq 0
$$

then $K=\overline{\mathbf{A}+\mathbf{B}}$. Thus the solution $\left(p_{n}\right)_{n=1}^{\infty}=\mathbf{p}=G_{K}(t) \stackrel{o}{\mathbf{p}}=$ $\left(G_{K_{n}}(t) \stackrel{o}{p_{n}}\right)_{n=1}^{\infty}$ satisfies

$$
\frac{d}{d t} \sum_{n=1}^{\infty} \int_{\mathbb{R}^{3}} G_{K_{n}}(t) \stackrel{o}{p_{n}}(x, n) n d x=\frac{d}{d t}\left\|G_{K_{n}}(t) \stackrel{o}{p_{n}}\right\|=0
$$

and for any ${\stackrel{o}{\mathbf{p}_{n}}}_{n}=(\stackrel{o}{p})_{n=1}^{\infty} \in D(K)_{+}$. In other words, the semigroup $\left(G_{K}(t)\right)_{t \geq 0}$ is honest.

Proof. The method we employ is analogous to that used in [2, Theorem 6.22]. Assume that for any $\mathbf{p}=\left(p_{n}\right)_{n=1}^{\infty} \in\left(\mathscr{X}_{1}\right)_{+}$ such that $-\mathbf{A p}+\mathbf{B} \mathbf{p} \in \mathscr{X}_{1}$ the inequality (31) holds; then we have

$$
\begin{aligned}
&-\int_{\mathbb{R}^{3}} \sum_{n=1}^{\infty} n a(y, n) g(y, n) d y \\
&+\int_{\mathbb{R}^{3}}\left(\sum_{n=1}^{\infty} n \sum_{m=n+1}^{\infty} \int_{\mathbb{R}^{3}} a(y, m) b_{n, m}(y) h(x, n, m, y) g\right. \\
&\times(y, m) d y) d x \geq 0 .
\end{aligned}
$$

By Proposition 2 and [2, Theorems 6.13 and 6.22], it is enough to show, for each $n \in \mathbb{N}$, that any $f_{n}(x)=f(x, n) \in F_{n+}$ such that $-f_{n}+\mathscr{B}_{n} L f_{n} \in X_{x}$; the following inequality holds:

$$
\begin{aligned}
& \sum_{n=1}^{\infty} \int_{\mathbb{R}^{3}}\left[L f_{n}\right](x) n d x \\
& \quad+\sum_{n=1}^{\infty} \int_{\mathbb{R}^{3}}\left(-f_{n}(x)+\left[\mathscr{B}_{n} L f_{n}\right](x)\right) n d x \geq 0,
\end{aligned}
$$

where $F_{n}:=\left\{\psi \in \Theta ; \quad\left(1+a_{n}\right)^{-1} \psi \in X_{x}\right\}, L:\left(F_{n}\right)_{+} \rightarrow X_{x}$ is defined such that $L f_{n}:=\left(1+a_{n}\right)^{-1} f_{n}$ and $\mathscr{B}_{n}$ is given by $\mathfrak{B} \mathbf{p}=\left(\mathscr{B}_{n} p_{n}\right)_{n=1}^{\infty}$ defined in (11). Now let $f_{n} \in F_{n+}$ such that $-f_{n}+B_{n} L f_{n} \in X_{x}$; let us set $g_{n}:=L f_{n}$; it is clear that $g_{n} \in$ $\left(X_{x}\right)_{+}$. Furthermore

$$
\begin{aligned}
-a_{n} g_{n}+B_{n} g_{n} & =-a_{n} L f_{n}+B_{n} L f_{n} \\
& =L f_{n}+\left(-f_{n}+B_{n} L f_{n}\right) \in X_{x} .
\end{aligned}
$$


Since $g_{n}$ satisfies the assumption, we have that

$$
\begin{aligned}
\sum_{n=1}^{\infty} \int_{\mathbb{R}^{3}}\left[L f_{n}\right](x) n d x & \\
& +\sum_{n=1}^{\infty} \int_{\mathbb{R}^{3}}\left(-f(x, n)+\left[B_{n} L f_{n}\right](x)\right) n d x \\
= & \sum_{n=1}^{\infty} \int_{\mathbb{R}^{3}}\left(g_{n}(x)-\left(1+a_{n}(x)\right) g_{n}(x)\right. \\
= & \left.+\left[B_{n} g_{n}\right](x)\right) n d x \\
& \sum_{n=1}^{\infty} \int_{\mathbb{R}^{3}}\left(-a_{n}(x) g_{n}(x)+\left[B_{n} g_{n}\right](x)\right) n d x \geq 0 .
\end{aligned}
$$

The second part of the lemma follows from (30).

This lemma allows us to state the following theorem.

Theorem 6. Assume that condition (1) is satisfied for almost all $(x, n) \in \mathbb{R}^{3} \times \mathbb{N}$, that is, $\theta_{1} \alpha_{n} \leq a_{n}(x) \leq \theta_{2} \alpha_{n}$; then the semigroup $\left(G_{K}(t)\right)_{t \geq 0}$ is honest.

Proof. The proof is based on Theorem 6.13 of [2]. Let $\mathbf{p}=$ $\left(p_{n}\right)_{n=1}^{\infty} \in\left(\mathscr{X}_{1}\right)_{+}$, by $(1)$; we have each $a_{n} p_{n} \in X_{x}:=$ $L_{1}\left(\mathbb{R}^{3}, d x\right)$. Because $-\mathbf{A p}+\mathbf{B} \mathbf{p} \in \mathscr{X}_{1}$, we also have $\mathscr{B}_{n} p_{n} \in$ $X_{x}:=L_{1}\left(\mathbb{R}^{3}, d x\right)$. So, making use of Lemma 5 , it is enough to prove that the inequality $\int_{\Lambda}(-\mathbf{A p}+\mathbf{B} \mathbf{p}) d \mu d \varsigma \geq 0$ is satisfied. We have

$$
\begin{aligned}
& \int_{\Lambda}(-\mathbf{A p}+\mathbf{B p}) d \mu d \varsigma \\
&= \sum_{n=1}^{\infty} \int_{\mathbb{R}^{3}}\left(\left(-a(x, n) p_{n}(x, n)+\left[\mathscr{B}_{n} p_{n}\right](x)\right) n d x\right) d x \\
&=\lim _{N \rightarrow \infty}\left(\sum_{n=1}^{N} \int_{\mathbb{R}^{3}}-a(x, n) p_{n}(x) n d x\right. \\
&\left.\quad+\sum_{n=1}^{N} \int_{\mathbb{R}^{3}}\left[\mathscr{B}_{n} p_{n}\right](x) n d x\right) .
\end{aligned}
$$

Also by (6),

$$
\begin{aligned}
& \sum_{n=1}^{N} \int_{\mathbb{R}^{3}}\left[\mathscr{B}_{n} p_{n}\right](x) n d x \\
& =\sum_{n=1}^{N} n \sum_{m=n+1}^{\infty} \int_{\mathbb{R}^{3}} a(y, m) b_{n, m}(y) \\
& \quad \times\left(\int_{\mathbb{R}^{3}} h(x, n, m, y) d x\right) p_{m}(y) d y \\
& =\int_{\mathbb{R}^{3}}\left(\sum_{n=1}^{N} n \sum_{m=n+1}^{\infty} a(y, m) b_{n, m}(y) p_{m}(y)\right) d y .
\end{aligned}
$$

Furthermore by (4), for almost all $y \in \mathbb{R}^{3}$

$$
\begin{aligned}
& \sum_{n=1}^{N} n \sum_{m=n+1}^{\infty} a(y, m) b_{n, m}(y) p_{m}(y) \\
& \quad=W_{N}(y)+\sum_{m=1}^{N} \sum_{n=1}^{m-1} n a(y, m) b_{n, m}(y) p_{m}(y) \\
& \quad=W_{N}(y)+\sum_{m=1}^{N} m a(y, m) p_{m}(y),
\end{aligned}
$$

where $W_{N}(y)=\sum_{m=N+1}^{\infty} \sum_{n=1}^{N} n a(y, m) b_{n, m}(y) p_{m}(y) \geq 0$. Combining, for any $N>0$ we have

$$
\begin{aligned}
\sum_{n=1}^{N} \int_{\mathbb{R}^{3}} & \left(-a(x, n) p_{n}(x)+\left[\mathscr{B}_{n} p_{n}\right](x)\right) n d x \\
= & \sum_{n=1}^{N} \int_{\mathbb{R}^{3}}-[a p](x, n) n d x \\
& +\int_{\mathbb{R}^{3}}\left(W_{N}(y)+\sum_{m=1}^{N} m[a p](y, m)\right) d y \\
= & \int_{\mathbb{R}^{3}} W_{N}(y) d y \geq 0 .
\end{aligned}
$$

Therefore

$$
\begin{gathered}
\sum_{n=1}^{\infty} \int_{\mathbb{R}^{3}}\left(-a(x, n) p_{n}(x)+\left[\mathscr{B}_{n} p_{n}\right](x)\right) n d x \\
=\lim _{N \rightarrow \infty} \int_{\mathbb{R}^{3}} W_{N}(y) d y \geq 0 .
\end{gathered}
$$

\section{Concluding Remarks and Discussion}

We have used a discrete model describing multiple fragmentation processes to show the honesty (conservativeness) of the system. The originality was to combine a discrete model with a nonlocal multiple fragmentation process where each fragmentation rate $a_{n}, n \in \mathbb{N}$, is size and position dependent and where new particles are spatially randomly distributed according to the given probabilistic law $h(\cdot, n, m, y)$ defined in (6). The main result here is the conservativeness in the nonlocal discrete fragmentation model described above. The method of semigroups with a parameter described in Section 3.1 together with Theorem 6 helps us to show that when each fragmentation rate $a_{n}, n \in \mathbb{N}$ is bounded by a size only dependent function, the spatial and random distribution of the particles has no influence on the conservativeness of the system. In other words nonlocal discrete models with each $a_{n}(x)$ bounded as $|x|$ approaches infinity always behave like local models and therefore are conservative provided that the fragmentation rate $a_{n}$ is bounded as $n$ approaches zero. However there is a major problem that arises when, in the discrete case, each fragmentation rate $a_{n}(x)$ becomes infinite as $|x|$ is close to infinity. This could be the subject of future investigations. 


\section{References}

[1] J. Banasiak and W. Lamb, “The discrete fragmentation equation: semigroups, compactness and asynchronous exponential growth," Kinetic and Related Models, vol. 5, no. 2, pp. 223-236, 2012.

[2] J. Banasiak and L. Arlotti, Perturbations of Positive Semigroups with Applications, Springer Monographs in Mathematics, Springer, London, UK, 2006.

[3] J. Banasiak and W. Lamb, "On the application of substochastic semigroup theory to fragmentation models with mass loss," Journal of Mathematical Analysis and Applications, vol. 284, no. 1, pp. 9-30, 2003.

[4] R. M. Ziff and E. D. McGrady, "The kinetics of cluster fragmentation and depolymerisation," Journal of Physics A, vol. 18, no. 15, pp. 3027-3037, 1985.

[5] C. R. Garibotti and G. Spiga, "Boltzmann equation for inelastic scattering," Journal of Physics A, vol. 27, no. 8, pp. 2709-2717, 1994.

[6] A. Majorana and C. Milazzo, "Space homogeneous solutions of the linear semiconductor Boltzmann equation," Journal of Mathematical Analysis and Applications, vol. 259, no. 2, pp. 609629, 2001

[7] J. Banasiak, "On an extension of Kato-Voigt perturbation theorem for substochastic semigroups and its applications," Taiwanese Journal of Mathematics, vol. 5, no. 1, pp. 169-191, 2001.

[8] W. Wagner, "Explosion phenomena in stochastic coagulationfragmentation models," The Annals of Applied Probability, vol. 15, no. 3, pp. 2081-2112, 2005.

[9] E. D. McGrady and R. M. Ziff, "'Shattering' transition in fragmentation," Physical Review Letters, vol. 58, no. 9, pp. 892895, 1987.

[10] B. Haas, "Loss of mass in deterministic and random fragmentations," Stochastic Processes and Their Applications, vol. 106, no. 2, pp. 245-277, 2003.

[11] J. Banasiak, "Kinetic-type models with diffusion: conservative and nonconservative solutions," Transport Theory and Statistical Physics, vol. 36, no. 1-3, pp. 43-65, 2007.

[12] K. Engel and R. Nagel, One-Parameter Semigroups for Linear Evolution Equations, Graduate Texts in Mathematics, Springer, Berlin, Germany, 2000.

[13] E. Hille and R. S. Phillips, Functional Analysis and Semigroups, vol. 31 of Colloquium Publications, American Mathematical Society, Providence, RI, USA, 1957. 


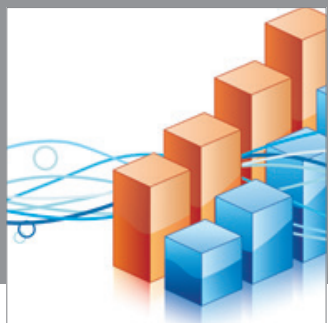

Advances in

Operations Research

mansans

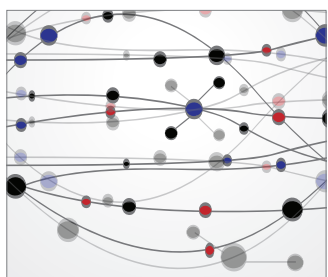

The Scientific World Journal
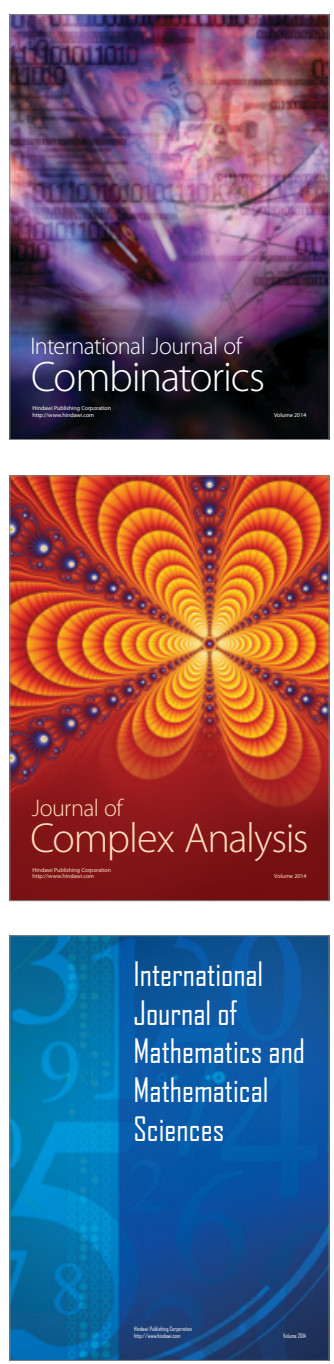
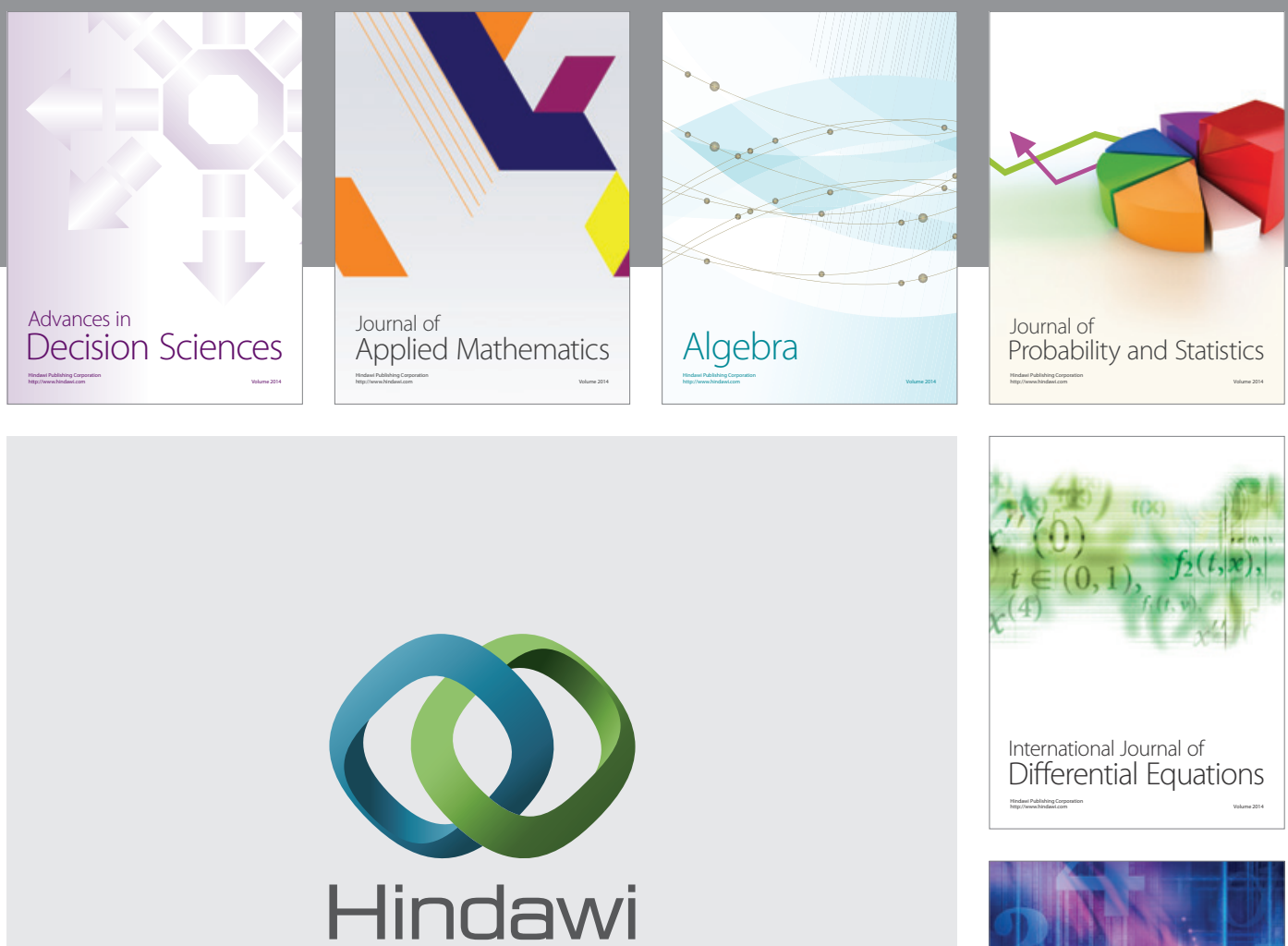

Submit your manuscripts at http://www.hindawi.com
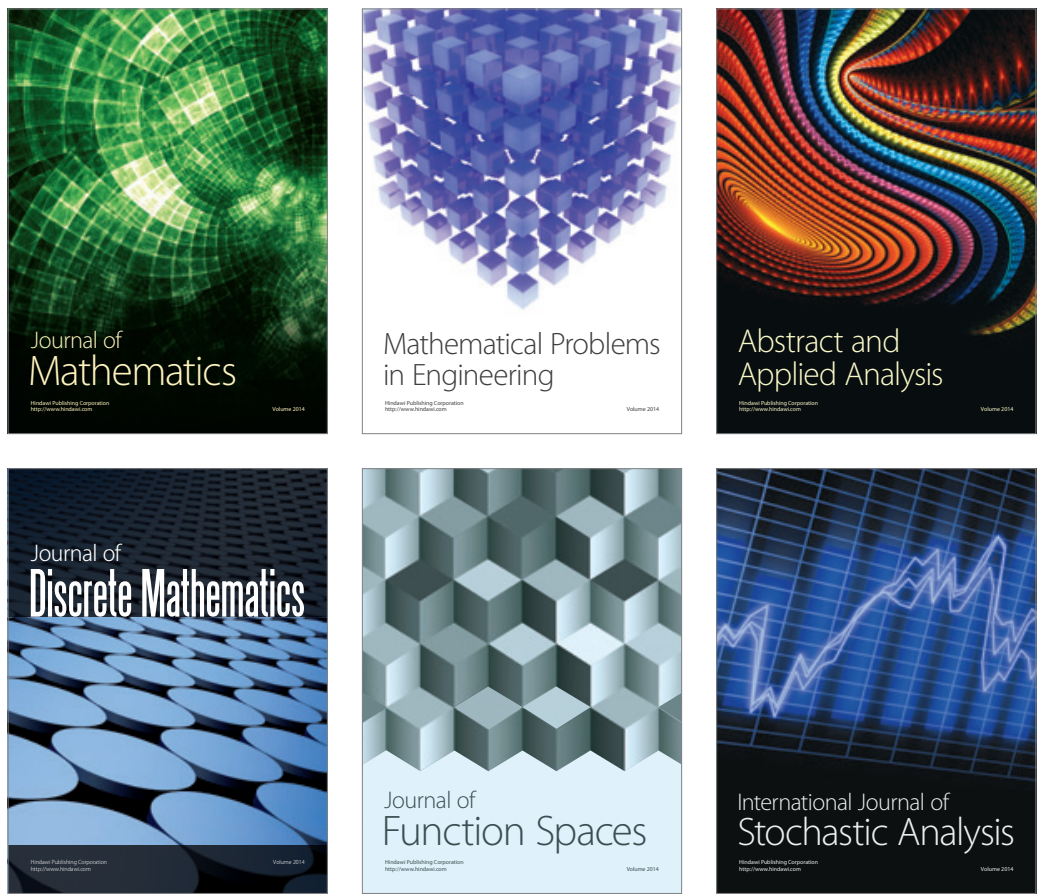

Journal of

Function Spaces

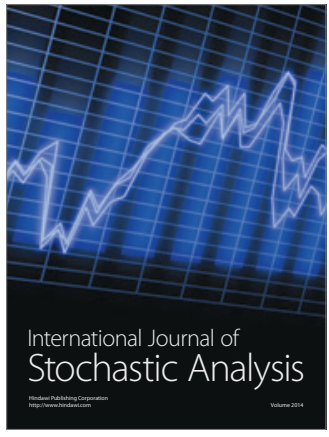

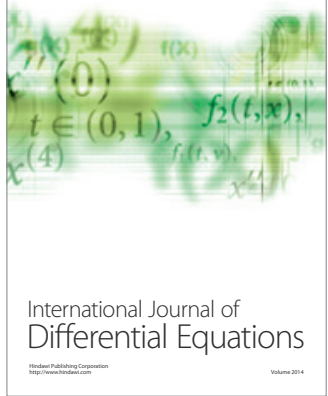
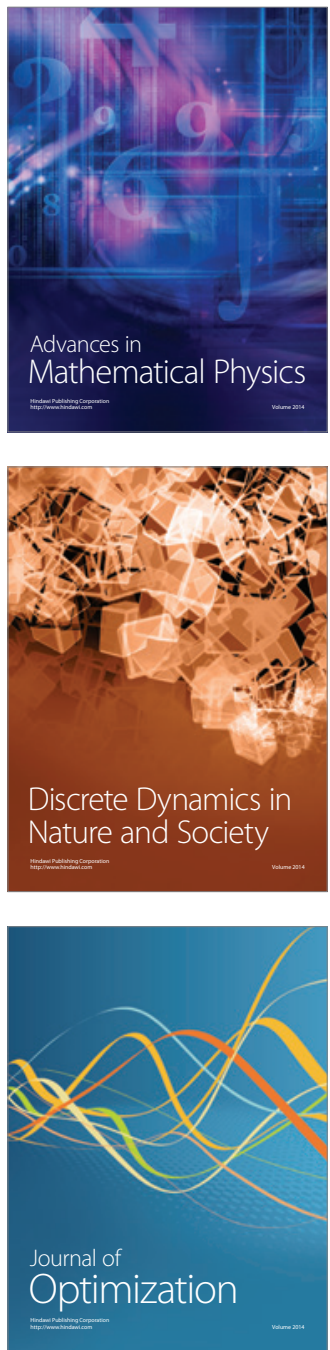